Ann. Biol. anim. Bioch. Biophys., I967, 7 (4), 39I-406.

\title{
ACTION DE LA PROGESTÉRONE ET DES STÉRÖ̈DES OVARIENS SUR LA SEGMENTATION DES EUFS CHEZ LA BREBIS
}

\author{
Suzanne WINTENBERGER-TORRES \\ avec la collaboration technique de Léone Rubin, Micheline Gérard, Aline Solari, C. Cornu \\ Station centrale de Physiologie animale, \\ Centre national de Recherches zootechniques, 78 -Jouy-en-Josas \\ Institut national de la Recherche agronomique
}

\section{SOMMAIRE}

On a étudié le rythme de segmentation des œufs prélevés sur des Brebis I à I I jours après l'ovulation. Après fixation, inclusion, coupe et coloration des œufs, le dénombrement des noyaux sur coupes histologiques sériées a été fait.

La vitesse de croissance est beaucoup plus élevée entre 8 et II jours qu'entre I et 7 jours. Elle a été également déterminée chez les œufs provenant de Brebis superovulées (la superovulation étant un moyen d'augmenter naturellement le taux de progestérone circulante) et chez les cufs issus de mères ayant eu des ovulations simples ou multiples, mais soumises à différents traitements de progestérone exogène. De I à 7 jours, l'œuf fécondé est insensible au niveau de progestérone auquel est soumis la mère. En revanche, de 8 à i I jours il est fortement stimulé par l'élévation du taux de progestérone. L'accélération maximum de développement enregistrée, est obtenue lorsqu'il y a superovulation et non avec des apports exogènes de progestérone, ce qui fait penser qu'en plus de la progestérone, des stéroïdes ovariens participeraient activement à cette stimulation.

\section{INTRODUCTION}

L'étude de la progression des œufs dans l'oviducte des Mammifères étudiés jusqu'à ce jour, comme la Souris, la Ratte, la Lapine, la Truie, la Brebis, la Vache, laisse apparaître qu'elle est sous la dépendance des niveaux d'œstrogène et de progestérone qui s'établissent après l'ovulation.

(1) Ce travail constitue une partie d'une thèse de Doctorat d'État (Sciences naturelles) présentée à la Faculté des Sciences de Paris le I 5 juin I 967 ( $\mathrm{n}^{\circ}$ Ao 1493 à l'enregistrement du Centre national de la Recherche scientifique). 
En modifiant le taux respectif de ces hormones, on agit sur la durée des différentes étapes qui caractérisent le transit, et par voie de conséquence sur la survie embryonnaire.

ADAms (I958) a montré en effet, chez la Lapine, que la rétention des œufs jusqu'à 4 jours dans les oviductes, qui peut être obtenue par injection de fortes quantités d'œstrogènes, arrête le développement des blastocystes. Par contre, l'accélération de la descente sous l'effet de faibles quantités d'œstrogène et l'arrivée précoce dans l'utérus, ne permettent d'observer le développement que d'une faible proportion d'œufs.

Dans ces conditions, l'utérus n'ayant pas atteint le stade de progestation correspondant à l'âge de l'œuf, ne peut en assurer la survie. On pensait que la désynchronisation entre l'âge de l'œuf et l'état de l'utérus était seule en cause pour expliquer la mortalité embryonnaire après accélération de la descente de l'œuf, sous 1'effet d'un déséquiilibre hormonal. Or, les travaux de GREENWALD (I957, I958) sont venus montrer que le traitement astrogénique après ovulation entraînait un amincissement de la zone albumineuse des oufs chez la Lapine, non seulement parce que les œufs séjournaient moins longtemps dans l'oviducte, mais aussi parce que les œstrogènes empêchaient la décharge de mucine provoquée dans l'épithélium tubaire par la progestérone.

On avait donc une preuve de l'action directe de l'équilibre hormonal sur la morphologie des œufs. Il restait à savoir si cette modification n'était pas sans influencer le développement ultérieur des œufs. La transplantation (GREENWAI,D, I962) de blastocystes de 3 jours à zone albumineuse amincie, en même temps que des blastocystes normaux, respectivement dans chaque corne utérine de Lapines receveuses, à œstrus synchronisé avec les donneuses, aboutissait à 29,2 p. roo d'implantations tandis que pour les blastocystes normaux, la proportion d'implantations était de 68,9 p. roo.

Actuellement, l'étude du mode d'action des contraceptifs a remis en question le problème de leur effet sur la motilité de l'oviducte et de l'utérus et de leur action propre sur le début du développement embryonnaire. Ceux qui n'agissent pas comme anti-implantatoires perturbent le transit des oufs par rétention ou accélération à travers 1'oviducte. Seul Davidson (Ig65) attribue au clomiphène (contraceptif chez le Rat) un pouvoir cytolytique direct sur l'œuf.

Dans nos précédents travaux (WNTENBERGER-ToRRìs S., I96I), nous avions constaté la nécessité de l'établissement d'un équilibre œestrogène/progestérone, bien déterminé, permettant la progression des œufs chez la Brebis, par action sur la motilité de 1'oviducte. En fonction des problèmes posés chez d'autres espèces, il nous a paru intéressant de suivre le développement des cufs chez des Brebis normales et soumises à différents traitements hormonaux.

Comme nous avions constaté une accélération du transport dans les cas de superovulations (WINTENBERGER S., I953), nous nous sommes attachés tout particulièrement à l'étude de la segmentation en présence de niveaux élevés de stéroïdes ovariens,

On sait en effet, depuis Short (I96r), Stormshak et al. (I963), que la superovulation et l'augmentation du nombre de corps jaunes qu'elle entraîne, provoque une élévation importante, principalement de la progestérone dans le sang. 
C'est pourquoi, dans une seconde série d'expériences, nous avons étudié l'effet de l'augmentation provoquée de la progestéronémie sur la vitesse de segmentation d'œufs issus d'ovulation simple et de superovulation.

\section{MATÉRIEL, ET MÉTHODES}

Afin de déterminer aussi précisément que possible l'âge des ceufs dont on veut étudier la segmentation, on déclenche l'ovulation $24 \mathrm{~h}$ après le début des chaleurs, par une injection intraveineuse en début d'œstrus de 400 UI d'hormone gonadotrope d'origine sérique (P. M. S. Organon). Trois accouplements sont provoqués pendant l'œstrus.

On déclenche une superovulation par une injection sous-cutanée, au $13^{e}$ jour $1 / 2$ ou $14^{e}$ jour du cycle de 2000 UI de gonadotropine d'origine sérique (P. M. S. Organon). Les ovulations sont régularisées comme précédemment.

L'apport exogène de progestérone est fait sous forme de solution huileuse injectée par voie sous-cutanée et donnée journellement de j 2 à $\mathrm{j}$ I I ( $\mathrm{j}$ ○ étant le moment de l'ovulation). La dose journalière a été :

a) dans le cas d'ovulation simple de $20 \mathrm{mg}$,

b) dans les cas de superovulation de 10,20 ou $40 \mathrm{mg}$.

Les Brebis sont abattues toutes les $24 \mathrm{~h}$, entre I et I I jours après l'ovulation.

Les cufs ou les blastocystes sont récupérés après perfusion des oviductes ou des cornes puis fixés, colorés, coupés à ro $\mu$. Après coloration à l'hématoxyline/eosine, leur développement est estimé par dénombrement de tous les noyaux.

\section{RÉSUL,TATS}

\section{Etat de segmentation des ceufs "témoins"}

Chez les Brebis n'ayant subi aucun traitement, la segmentation se déroule selon le schéma suivant:

L a première division peut être terminée $24 \mathrm{~h}$ après l'ovulation, la deuxième lui succéder dès $36 \mathrm{~h}$. Le stade 8 est atteint à $48 \mathrm{~h}$ et le stade I2 à $72 \mathrm{~h}$. Ces temps représentent des délais minimum qui peuvent être allongés ; en effet, nous avons observé des stades 2 à $3 \mathrm{I} \mathrm{h}$, des stades 8 à $72 \mathrm{~h}$. La division des premiers blastomères n'est pas toujours synchrone puisqu'il existe des stades $3,6,7$, I2 et I4.

Le tableau I résume l'état de segmentation des œufs de I à 5 jours.

TABLEAU I

Nombre de blastomères des aufs "témoins " de 1 à 5 jours

\begin{tabular}{|c|c|c|c|c|}
\hline Age & Nombre de brebis & Nombre d'œufs & $\begin{array}{l}\text { Nombre moyen } \\
\text { de blastomères }\end{array}$ & Mini/Maxi (cell.) \\
\hline $\begin{array}{rl}24 & \mathrm{~h} \\
48 \mathrm{~h} \\
72 \mathrm{~h} \\
96 \mathrm{~h} \\
120 \mathrm{~h}\end{array}$ & $\begin{array}{l}5 \\
3 \\
5 \\
9 \\
7\end{array}$ & $\begin{array}{r}6 \\
6 \\
8 \\
13 \\
9\end{array}$ & $\begin{array}{rr} & 3 \\
\# & 7 \\
\# & 10 \\
\# & 40 \\
\# & 104\end{array}$ & $\begin{array}{c}2-6 \\
4-8 \\
8-12 \\
21-76 \\
44-149\end{array}$ \\
\hline
\end{tabular}

Annales de Biologie animale. - 967 . 
A partir du $6 \mathrm{e}$ jour, il faut tenir compte de la différenciation du bouton embryonnaire et du trophoblaste. Les résultats sont rapportés dans le tableau 2. ..

TABLEAU 2

Nombre de cellules des aufs " témoins" de 6 à 11 jours

\begin{tabular}{|c|c|c|c|c|c|c|c|c|}
\hline \multirow{2}{*}{$\begin{array}{l}\text { Age } \\
\text { ceufs }\end{array}$} & \multirow{2}{*}{$\begin{array}{c}\text { Nbre } \\
\text { brebis }\end{array}$} & \multirow{2}{*}{$\begin{array}{l}\text { Nbre } \\
\text { ceufs }\end{array}$} & \multicolumn{2}{|c|}{ Nombre total de cellules } & \multicolumn{2}{|c|}{ Trophoblaste } & \multicolumn{2}{|c|}{ Bouton embryonnaire } \\
\hline & & & moyenne & $\operatorname{mini} / \operatorname{maxi}$ & moyenne & $\operatorname{mini} / \operatorname{maxi}$ & moyenhe & $\operatorname{mini} / \operatorname{maxi}$ \\
\hline $6 \mathrm{j}$ & 6 & 9 & 211 & $138-308$ & 128 & $84-186$ & 71 & $38-122$ \\
\hline $7 \mathrm{j}$ & 6 & 7 & 419 & $192-651$ & 356 & $144-583$ & 61 & $45-91$ \\
\hline $8 \mathrm{j}$ & 6 & 9 & 373 & $250-543$ & 307 & $200-438$ & 66 & $35-105$ \\
\hline $9 \mathrm{j}$ & 6 & 8 & 663 & $231-1441$ & 584 & $164-1258$ & 79 & $38-183$ \\
\hline $10 \mathrm{j}$ & 7 & 10 & 1874 & $503-5900$ & 1863 & $438-414: 1$ & 155 & $64-305$ \\
\hline $11 \mathrm{j}$ & 11 & 16 & 10203 & $3296-28542$ & 9502 & $3041-26530$ & 695 & $255-2012$ \\
\hline
\end{tabular}

La croissance est régulière jusqu'à 7 jours, subit un léger fléchissement à 8 jours et repart de façon très accélérée de 9 à II jours.

En transformant en racine carrée les données relatives au nombre de noyaux $(\mathrm{N})$ on peut représenter le développement par deux droites de régression de formule :

$$
\begin{aligned}
& \mathrm{A}_{1} \mathrm{Y}_{1}=3, \mathrm{II} x-5,5 \\
& \mathrm{~B}_{1} \mathrm{Y}_{1}=2 \mathrm{I}, 54 x-\mathrm{I} 55,47
\end{aligned}
$$

Le segment $\mathrm{A}$ correspond à la période de $\mathrm{I}-7$ jours et le segment $\mathrm{B}$ à celle de 8 - Ir jours, le coefficient de cette droite très significativement supérieur à la première témoigne de la croissance accélérée après 8 jours (fig. I).

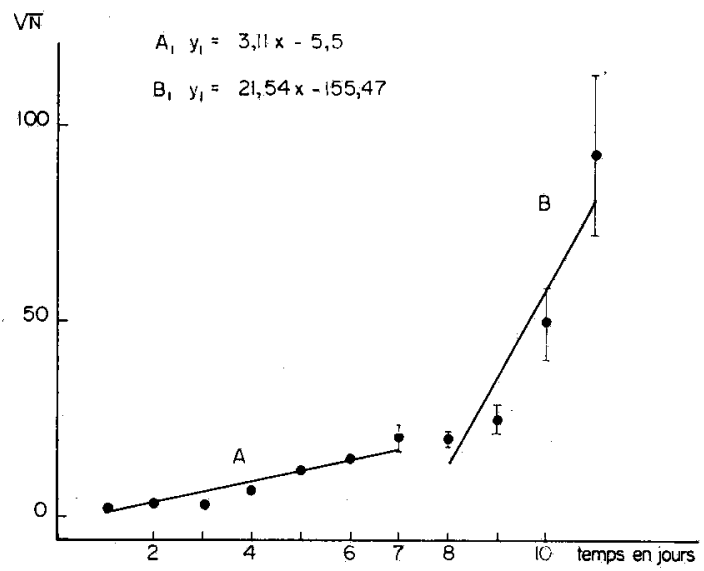

FIG. I, - État de segmentation des oufs "témoins"

$\mathrm{N}$ : nombre de noyaux comptés sur les coupes histologiques sériées des œufs.

Pour chaque point, l'ordonnée représente une valeur moyenne établie après numération de 6 à ı 6 œufs. Les écarts-types correspondants sont indiqués. Entre I et 6 jours, leur valeur est trop faible pour être représentée. 
Le trophoblaste qui constitue la partie la plus importante des blastocystes, a évidemment les mêmes caractéristiques de développement. Quant au bouton embryonnaire, son évolution, représentée sur la figure 2 , est proportionnellement beaucoup moins rapide que celle du trophoblaste.

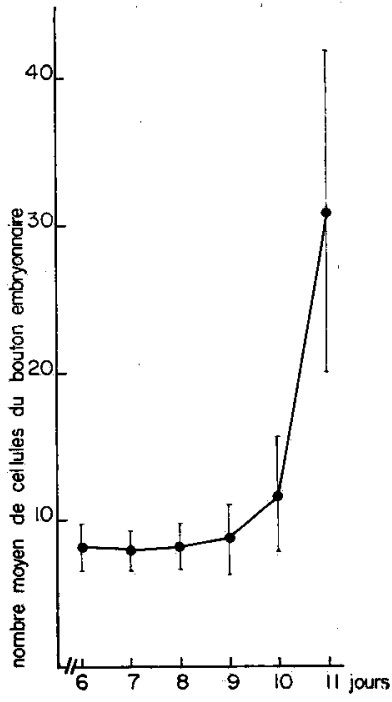

FIG. 2. - Nombre de cellules du bouton embryonnaire des oufs "témoins" de 6 a 11 jours.

Pour chaque point, l'ordonnée représente une moyenne établie à partir de 7 à 16 œufs.

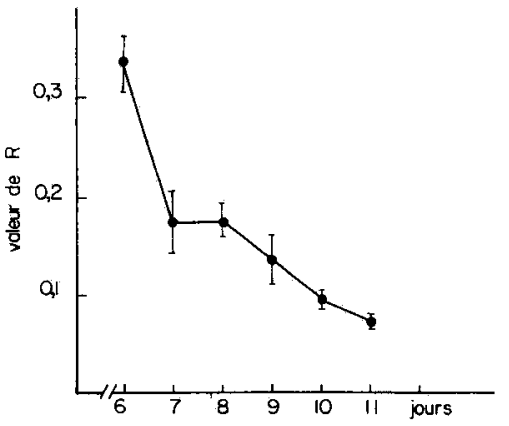

Fig. 3. - Evolution du rapport : Nombre moyen de cellules du bouton embryonnaire. Nombre moyen de cellules du blastocyste des oufs " témoins"

$\mathrm{Si}$ on fait le rapport ( $\mathrm{R})$ entre le nombre moyen : cellules du bouton embryonnaire/cellules du trophoblaste, à 6 jours, il est voisin de 0,35 à mesure que le blastocyste croît, il décroît pour tomber à 0,05 à II jours (fig. 3).

\section{Etat de segmentation des ceufs "superovulés"}

L'injection d'hormone gonadostimulante permet d'obtenir environ 5 à ro oufs par Brebis. Leur état de division jusqu'à 5 jours est représenté dans le tableau 3 .

\section{TABLEAU 3}

Nombre de blastomères des oufs "superovulés " de 1 à 5 jours

\begin{tabular}{|c|c|c|c|c|c|}
\hline \multirow{2}{*}{ Age des œufs } & \multirow{2}{*}{\multicolumn{2}{|c|}{ Nombre de brebis }} & \multirow{2}{*}{ Nombre d'œufs } & \multicolumn{2}{|c|}{ Nombre total de cellules } \\
\hline & & & & moyenne & $\min i / \operatorname{maxi}$ \\
\hline 1 jour & & 5 & 28 & \# & $2-6$ \\
\hline 2 jours & & 5 & 17 & \# & $2-8$ \\
\hline 3 jours & & 5 & 32 & & $2-25$ \\
\hline 4 jours & $\div$ & 5 & 45 & \# 32 & $7-78$ \\
\hline 5 jours & & 9 & 39 & \# 141 & $29-465$ \\
\hline
\end{tabular}


Comparés aux œufs " témoins ", leur rythme de segmentation pendant cette période n'est pas différent.

Par contre, entre 6 et II jours, la vitesse de division est non seulement supérieure à la période de I- 5 jours, mais aussi très nettement accélérée, surtout à partir de 8 jours, par rapport à celle enregistrée chez les œufs “ témoins » de même âge (tabl. 4).

TABLEAU 4

Nombre de cellules des coufs "superovulés " de 6 à 11 jours

\begin{tabular}{|c|c|c|c|c|c|c|c|c|}
\hline \multirow{2}{*}{$\begin{array}{l}\text { Age } \\
\text { œufs }\end{array}$} & \multirow{2}{*}{$\begin{array}{l}\text { Nbre } \\
\text { brebis }\end{array}$} & \multirow{2}{*}{$\begin{array}{l}\text { Nbre } \\
\text { œufs }\end{array}$} & \multicolumn{2}{|c|}{ Nombre total de cellules } & \multicolumn{2}{|c|}{ Trophoblaste } & \multicolumn{2}{|c|}{ Bouton embryonnaire } \\
\hline & & & moyenne & $\operatorname{mini} / \operatorname{maxi}$ & moyenne & $\operatorname{mini} / \operatorname{maxi}$ & moyenne & $\operatorname{mini} / \operatorname{maxi}$ \\
\hline $6 \mathrm{j}$ & 8 & 46 & 179 & $68-307$ & 142 & $60-262$ & 53 & $15-100$ \\
\hline $7 \mathrm{j}$ & 12 & 41 & 341 & $80-1763$ & 282 & $49-1665$ & 66 & $27-111$ \\
\hline $8 \mathrm{j}$ & 8 & 29 & 872 & 223-1938 & 790 & $197-1797$ & 90 & $26-213$ \\
\hline $9 \mathrm{j}$ & 5 & 26 & 5113 & $1326-15035$ & 4832 & $1171-14618$ & 277 & $49-544$ \\
\hline $10 \mathfrak{j}$ & 2 & 12 & 74324 & $21347-205102$ & 72643 & $13442-201757$ & 1673 & $951-3245$ \\
\hline $11 \mathrm{j}$ & 4 & 16 & 81737 & $15417-254760$ & 80063 & $14192-253465$ & 1674 & $943-3187$ \\
\hline
\end{tabular}

En effectuant la transformation en racine carrée de ces données on construit deux droites de régression de formule :

$$
\begin{aligned}
& \mathrm{A}_{2} \mathrm{Y}_{2}=3,22 x-5,34 \\
& \mathrm{~B}_{2} \mathrm{Y}_{2}=82 x-637
\end{aligned}
$$

qui correspondent respectivement aux oufs âgés de $I$ à 7 jours et de 8 à Ir jours (fig. 4). Par rapport aux observations faites sur des œufs issus d'ovulations normales, on constate que les coefficients de régression des droites $\mathrm{A}\left(\mathrm{Y}_{1}=3, \mathrm{II} x-5,5\right.$ et $\left.\mathrm{Y}_{2}=3,22 x-5,34\right)$ ne sont pas significativement différents.

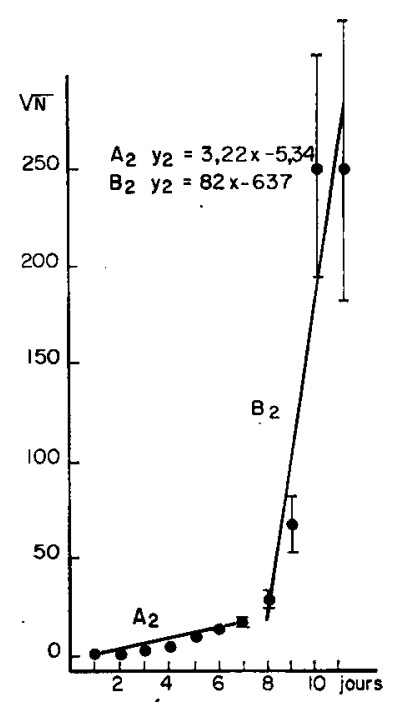

FIG. 4. - Etat de segmentation des oufs a superovulés "

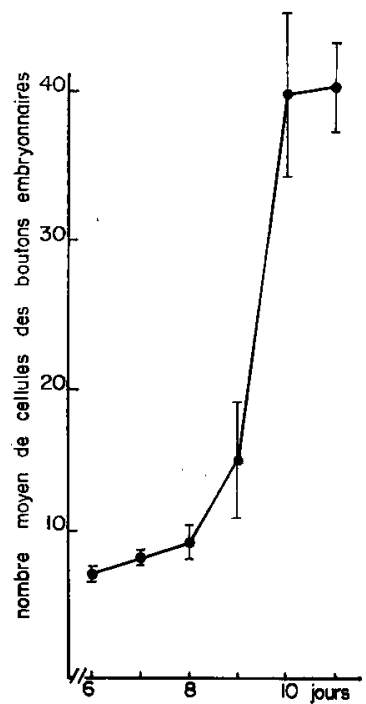

Fig. 5. - Nombre moyen de cellules des boutons em-

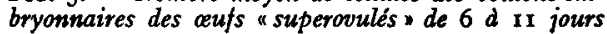


Par contre, si on compare les coefficients de régression des segments de droites $B$, des deux séries d'observations $\left(\mathrm{Y}_{1}=2 \mathrm{I}, 54 x-155,47\right.$ et $\left.\mathrm{Y}_{2}=82 x-637\right)$ ils sont significativement différents et très supérieur dans les cas de superovulation. L'accélération de la segmentation qui se manifeste ainsi à partir de 8 jours est considérable, certains blastocystes "superovulés " présentant en effet jusqu'à 8 fois plus de cellules que les blastocystes " normaux ".

Le développement des blastocystes issus de superovulation n'en reste pas moins harmonieux. La courbe de croissance du bouton embryonnaire est exprimée sur la figure 5. Les croissances relatives du trophoblaste et du bouton embryonnaire évoluent selon un rapport qui décroît progressivement de 0,30 à 0,005 en passant à 9 jours, par la valeur 0,05 trouvée à II jours chez les Brebis «normales " (fig. 6).

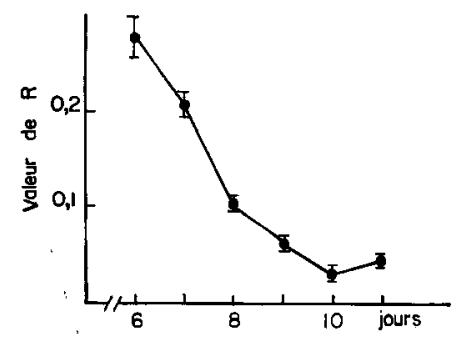

Fig. 6. - Évolution du rapport: Nombre moyen de cellules du bouton embryonnaire. Nombre moyen de cellules du blastocyste des eufs "superovulés"

\section{Vitesse de segmentation des ceufs après action de la progestérone}

Dans les différents groupes expérimentaux, les œufs sont prélevés à 3, 5, 6, 8 et II jours après l'ovulation.

a) Après ovulation normale et injection de $20 \mathrm{mg}$ de progestérone de $j 2$ à $j 11$.

Le tableau 5 donne l'état de division correspondant à ces différentes périodes. Après transformation en racine carrée des résultats, on construit deux droites de

TABLEAU 5

Nombre de cellules des cufs de 3 à 11 jours après traitement de $20 \mathrm{mg}$ de progestérone

\begin{tabular}{|c|c|c|c|c|c|c|c|c|}
\hline \multirow{2}{*}{$\begin{array}{l}\text { Age } \\
\text { œufs }\end{array}$} & \multirow{2}{*}{$\begin{array}{l}\text { Nbre } \\
\text { brebis }\end{array}$} & \multirow{2}{*}{$\begin{array}{l}\text { Nbre } \\
\text { œufs }\end{array}$} & \multicolumn{2}{|c|}{ Nombre total de cellules } & \multicolumn{2}{|c|}{ Trophoblaste } & \multicolumn{2}{|c|}{ Bouton embryonnaire } \\
\hline & & & moyenne & $\operatorname{mini} / \operatorname{maxi}$ & moyenne & $\operatorname{mini} / \operatorname{maxi}$ & moyenne & $\operatorname{mini} / \operatorname{maxi}$ \\
\hline $3 \mathrm{j}$ & 7 & 8 & \# 13 & $10-17$ & & & & \\
\hline $5 j$ & 8 & 10 & \# 102 & $60-151$ & & & & \\
\hline 6 & 8 & 12 & \# 134 & 139-164 & 90 & $64-116$ & 53 & $24-75$ \\
\hline 8 & 8 & 9 & 830 & 403-1 607 & 694 & 280-1 455 & 136 & $49-238$ \\
\hline $11 j$ & 6 & 8 & 21761 & 5 829-38 844 & 20816 & $5652-37487$ & 932 & $177-1863$ \\
\hline
\end{tabular}


régression qui sont comme précédemment, pour les périodes $I-7$ jours $\left(A_{3}\right)$ et $8-$ Ir jours $\left(B_{3}\right)$, et de formule :

$$
\begin{aligned}
& \mathrm{A}_{3} \mathrm{Y}_{3}=2,65 x-4 \\
& \mathrm{~B}_{3} \mathrm{Y}_{3}=38,66-28 \mathrm{I}, 25
\end{aligned}
$$

et qui sont représentées sur la figure 7 .

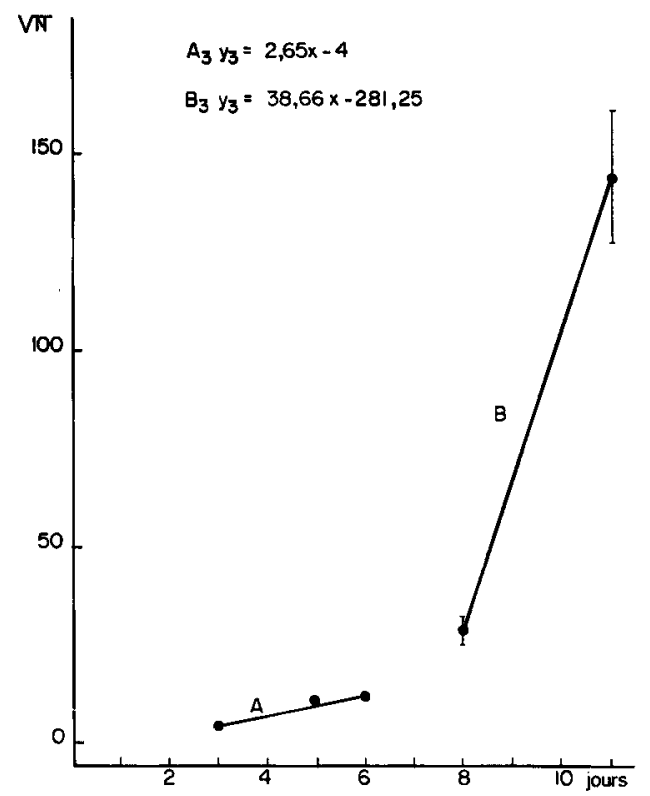

FiG. 7. - Étal de segmentation des oufs après traitement de $20 \mathrm{mg}$ de progestérone.

Comparée aux formules obtenues à partir des œufs témoins $\left(A_{1} Y_{1}=3, \operatorname{II} x-5,5\right.$ et $\left.B_{1} Y_{1}=21,54 x-155,47\right)$, la droite $A$ n'a pas un coefficient de régression significativement différent.

Par contre, si on compare les droites $B_{1}$ et $B_{3}$, on obtient après le traitement de progestérone, une nette augmentation : $x=38,66$ au lieu de 21,54 . L'injection journalière de $20 \mathrm{mg}$ de progestérone a une action sensible à partir du $8 \mathrm{e}$ jour. Le coefficient de régression n'atteint cependant pas celui de 82 obtenu dans le cas de superovulation.

b) Effet du traitement progestéronique après ovulation multiple.

Cette action stimulante de la progestérone sur la segmentation des cufs " témoins " nous a conduit à rechercher les limites d'action de la progestérone en essayant de cumuler un apport exogène et une hypersécrétion naturelle par superovulation.

a) Traitement journalier de $10 \mathrm{mg}$ de progestérone de $j 2$ à $j 11$.

Le tableau 6 donne les résultats des numérations effectuées sur les coupes histologiques des œufs recueillis de 3 à II jours après l'ovulation. Les formules des droites de régression représentées sur la figure $8 b$, sont :

$$
\begin{aligned}
& \mathrm{A}_{4} \mathrm{Y}_{4}=2,76 x-3,72 \\
& \mathbf{B}_{4} \mathrm{Y}_{4}=6 \mathrm{I}, 86 x-46 \mathrm{I}, 2
\end{aligned}
$$




\section{TABLEAU 6}

Nombre des cellules des oufs "superooulés" après traitement de $10 \mathrm{mg}$ de progestérone

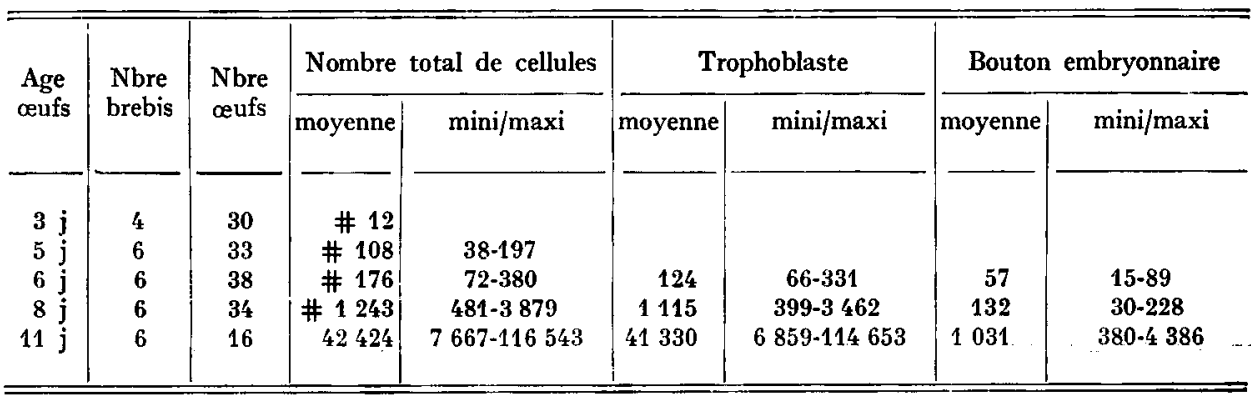
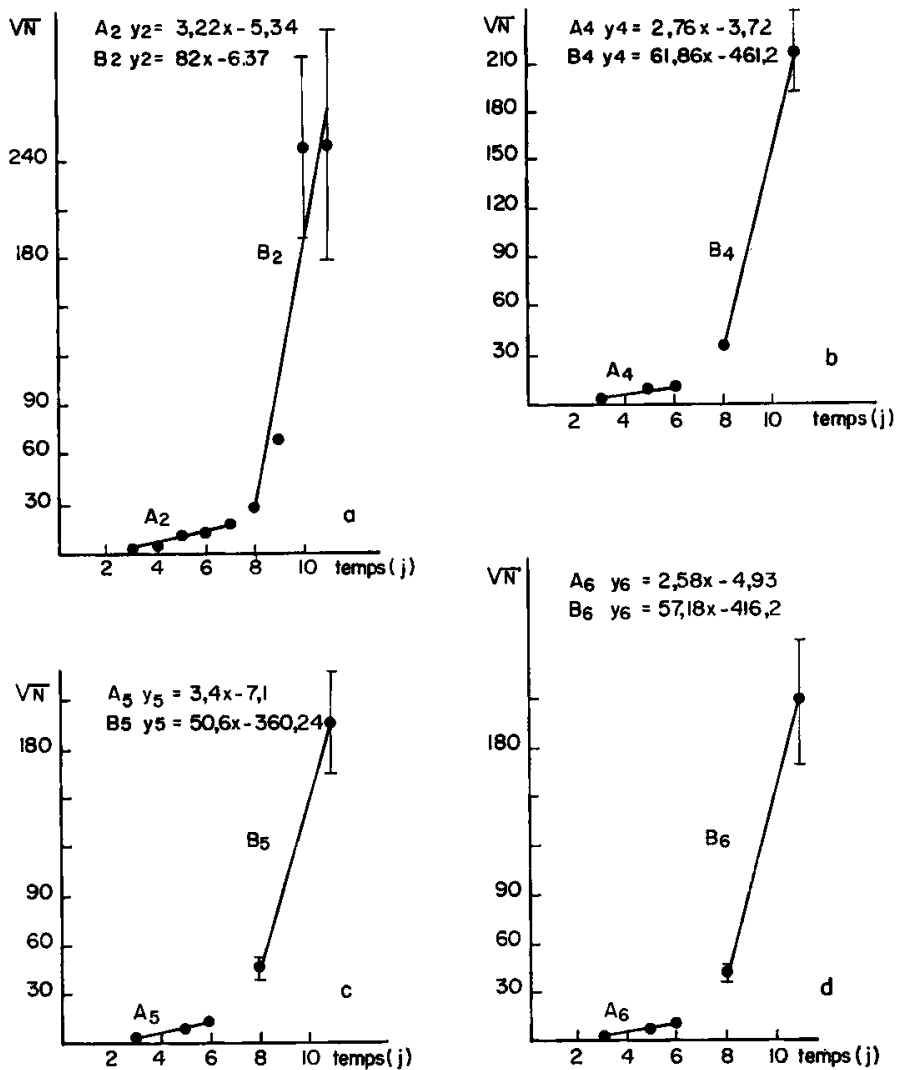

FIG. 8. - Etat de segmentation des aufs
a) superovulés (rappel),
b) avec to $\mathrm{mg}$ de progestérone,
c) avec $20 \mathrm{mg}$ de progestérone,
d) avec $40 \mathrm{mg}$ de progestéronc. 
3) Traitement journalier de $20 \mathrm{mg}$ de progestérone.

Le tableau 7 donne les résultats obtenus avec ce traitement et les droites de régression représentées sur la figure $8 c$, ont pour formule :

$$
\begin{aligned}
& A_{5} Y_{5}=3,4 x-7, \mathbf{I} \\
& B_{5} Y_{5}=50,6 x-360,24
\end{aligned}
$$

\section{TABLEAU 7}

Nombre de cellules des aufs "superovulés"

\begin{tabular}{|c|c|c|c|c|c|c|c|c|}
\hline \multirow{2}{*}{$\begin{array}{l}\text { Age } \\
\text { œufs }\end{array}$} & \multirow{2}{*}{$\begin{array}{l}\text { Nbre } \\
\text { brebis }\end{array}$} & \multirow{2}{*}{$\begin{array}{l}\text { Nbre } \\
\text { œufs }\end{array}$} & \multicolumn{2}{|c|}{ Nombre total de cellules } & \multicolumn{2}{|c|}{ Trophoblaste } & \multicolumn{2}{|c|}{ Bouton embryonnaire } \\
\hline & & & moyenne & $\operatorname{mini} / \operatorname{maxi}$ & moyenne & $\operatorname{mini} / \operatorname{maxi}$ & moyenne & $\operatorname{mini} / \operatorname{maxi}$ \\
\hline $3 \mathbf{j}$ & 9 & 25 & \# 19 & $9-43$ & & & & \\
\hline 5 & 6 & 29 & \# 125 & $50-210$ & & & & \\
\hline 6 & 7 & 22 & 151 & $71-391$ & $\# 121$ & $58-342$ & \# 38 & $21-71$ \\
\hline 8 & 5 & 26 & 1016 & $497-2803$ & 900 & $435-2618$ & \# 115 & $42-204$ \\
\hline $11 \mathfrak{j}$ & 6 & 13 & 50344 & $30020-111535$ & 47873 & $27452-108132$ & 2470 & $1686-4740$ \\
\hline
\end{tabular}
après action de $20 \mathrm{mg}$ de progestérone par jour

r) Traitement de $40 \mathrm{mg}$ de progestérone (tabl. 8).

Les droites de régression correspondantes représentées sur la figure $8 d$, sont de formules :

$$
\begin{aligned}
& \mathrm{A}_{6} \mathrm{Y}_{6}=2,58 x-4,93 \\
& \mathrm{~B}_{6} \mathrm{Y}_{6}=57, \mathrm{I} 8 x-4^{\mathrm{I} 6,2}
\end{aligned}
$$

\begin{tabular}{|c|c|c|c|c|c|c|c|c|}
\hline \multirow{2}{*}{$\begin{array}{l}\text { Age } \\
\text { œufs }\end{array}$} & \multirow{2}{*}{$\begin{array}{l}\text { Nbre } \\
\text { brebis }\end{array}$} & \multirow{2}{*}{$\begin{array}{l}\text { Nbre } \\
\text { cufs }\end{array}$} & \multicolumn{2}{|c|}{ Nombre total de cellules } & \multicolumn{2}{|c|}{ Trophoblaste } & \multicolumn{2}{|c|}{ Bouton embryonnaire } \\
\hline & & & moyenne & $\operatorname{mini} / \operatorname{maxi}$ & moyenne & $\operatorname{mini} / \operatorname{maxi}$ & moyenne & $\min i / \operatorname{maxi}$ \\
\hline $3 \mathrm{j}$ & 6 & 20 & $\# 11$ & $4-21$ & & & & \\
\hline $5 j$ & 7 & 36 & $\# 60$ & $13-173$ & & & & \\
\hline 6 & 4 & 28 & \# 128 & $76-224$ & $\# 90$ & $57-181$ & $\# 41$ & $33-86$ \\
\hline $8 j$ & 5 & 24 & \# 1426 & $399-3362$ & $\mid \begin{array}{l}\text { \# } 1259 \\
12\end{array}$ & $288-3167$ & \# 167 & $61+311$ \\
\hline $11 \mathrm{j}$ & 3 & 26 & 50903 & $46551-135934$ & 49215 & $6751-132596$ & 1755 & $320-3476$ \\
\hline
\end{tabular}

TABLEAU 8

Nombre de cellules des ceufs "superovulés" après action de $40 \mathrm{mg}$ de progestérone par jour 
Des doses variées de progestérone laissent inchangée la vitesse de segmentation jusqu'à 7 jours. L'accélération se produit à partir de 8 jours, par rapport aux œufs témoins, chaque fois qu'existe un niveau anormalement élevé de progestérone. Cependant, bien qu'il s'agisse d'œufs superovulés, l'apport de progestérone exogène ne permet plus d'obtenir l'accélération enregistrée avec une superovulation simple, sans supplémentation de progestérone.

Sur le tableau 9, on trouve la comparaison des variances calculées à $5,6,8$ et et II jours. Quand celles-ci sont homogènes, le test de Student ou loi normale a été employé, par contre, lorsqu'elles ne le sont pas, on utilise le test de Behrens et Fisher et les tables de Sukhatme.

\section{TABLEAU 9}

Comparaison des valeurs moyennes du nombre de cellules entre les aufs " superovulés "et " superovulés " + progestérone

\begin{tabular}{|c|c|c|c|c|c|c|}
\hline \multirow{2}{*}{ Groupes } & \multicolumn{2}{|c|}{5 jours } & \multicolumn{2}{|c|}{6 jours } & 8 jours & 11 jours \\
\hline & t calculé & $\mathrm{T}(5 \%)$ & $t$ calculé & T $(5 \%)$ & $t$ calculé & l calculé \\
\hline $\begin{array}{c}\text { So } \\
\text { so }+10 \mathrm{mg}\end{array}$ & \multicolumn{2}{|c|}{ NS } & \multicolumn{2}{|c|}{$\mathbf{S}$} & $\begin{array}{l}S \quad(2,85 \\
\text { a } 1 \%)\end{array}$ & $\begin{array}{r}4,40^{* *} \quad 2,174 \\
\text { HS }(3,00 \\
\text { à } 1 \%)\end{array}$ \\
\hline $\begin{array}{c}\text { So } \\
\text { SO }+20 \mathrm{mg}\end{array}$ & \multicolumn{2}{|c|}{ NS } & \multicolumn{2}{|c|}{ NS } & $\begin{array}{r}\text { HS }(2,98 \\
\text { à } 1 \%)\end{array}$ & $\mathrm{HS}$ \\
\hline $\begin{array}{c}\text { SO } \\
\text { So }+40 \mathrm{mg}\end{array}$ & $3,98^{* *}$ & 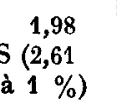 & $4,169^{* *}$ & 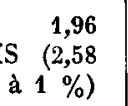 & $\begin{array}{rr}6,204^{* *} & 2,08 \\
\text { HS } & (2,80 \\
\text { à } 1 & \%)\end{array}$ & $\begin{array}{rr}22,17^{* *} & 2,112 \\
\text { HS }(2,85 \\
\text { à } 1 \%)\end{array}$ \\
\hline
\end{tabular}

SO $=$ superovulé

SO $+\mathrm{T}=$ superovulé + progestérone

$\mathrm{SO}+\mathrm{T}=$ superovulé + progestérone

$\mathrm{T}=$ valeur seuil à 5 p. 100 et à 1 p. $100 \quad\left({ }^{(} \bar{x}_{\mathrm{SO}} \bar{x}_{\mathrm{SO}+\mathrm{T}}=\right.$ écart type de la différence $)$

NS $=$ non significatif

$\mathrm{S}=$ significatif*

HS $=$ hautement significatif**

Comparés au cas de superovulation simple, les trois traitements présentent des variances significativement différentes, 1'homogénéité des résultats est particulièrement nette pour les points 8 et II jours. L'apport de progestérone dans les cas de superovulation freine 1'accélération du développement et n'a pas une action cumulée avec la superovulation.

Les variations que subissent les blastocystes dans leur croissance sous l'effet des différents traitements ne troublent pas l'harmonie entre le développement du bouton embryonnaire et le trophoblaste. I1 s'ensuit qu'une accélération de la vitesse de segmentation du blastocyste se traduit également par une accélération du développement 
du bouton embryonnaire. La figure 9 représente pour les quatre traitements à la progestérone les courbes de développement que suivent les boutons embryonnaires.

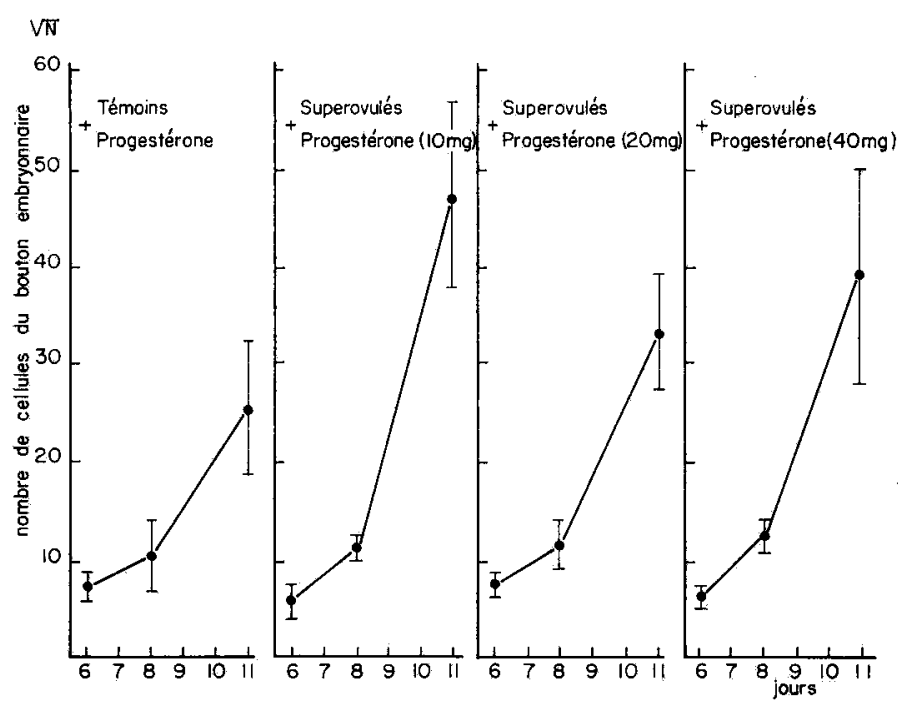

FIG. 9. - Nombre de cellules des boutons embryonnaires des cu/s soumis à differents traitements progestéroniques

\section{DÉGÉNÉRESCENCE}

Au cours de nos observations microscopiques, nous avons enregistré de nombreux cas de dégénérescence qui se manifestent de trois façons différentes :

- soit par une évolution picnotique des noyaux. Ce type de dégénérescence survient à tous les stades de développement étudiés.

- soit par des anomalies morphogénétiques qui se produisent vers 5-6 jours au moment de la formation du blastocyste et à I I jours pour les œufs témoins, c'est-à-dire quand le blastocyste s'allonge en vésicule filiforme ; chez les œufs dont l'évolution est plus rapide comme les œufs superovulés, cette période critique se trouve déplacée vers 9 jours.

- soit par un retard de segmentation. Nous avons considéré que les œufs présentant un retard de 2 jours ne pouvaient survivre. Les expériences de transplantations asynchrones ont montré, en effet, qu'un écart de plus de 48 h entre l'âge de l'œuf et le stade physiologique de l'utérus aboutissait à un échec.

On a établi pour chaque série expérimentale, le pourcentage de dégénérescence observé chez les œuts de même âge.

La figure Io $a$ représente la courbe obtenue avec les œufs «témoins ». L'augmentation de la dégénérescnece vers 6-7 jours correspond à la première période critique que traverse l'œuf au moment de la formation du blastocyste. Le deuxième maximum 
vers IO-I I jours correspond à la seconde période critique au moment de son allongement en une longue vésicule filiforme.

Pour les aufs "superovulés" (fig. Io $b$ ) on constate 1'existence d'un maximum vers 2 jours qui correspond aux œufs non fécondés ou anormaux dont la proportion est plus importante dans les cas de superovulation. Le deuxième maximum correspond, comme précédemment à la formation du blastocyste, et le troisième maximum qui coïncide avec l'allongement en vésicule filiforme est déplacé vers 9 jours car nous avons vu que ces blastocystes avaient un développement accéléré.

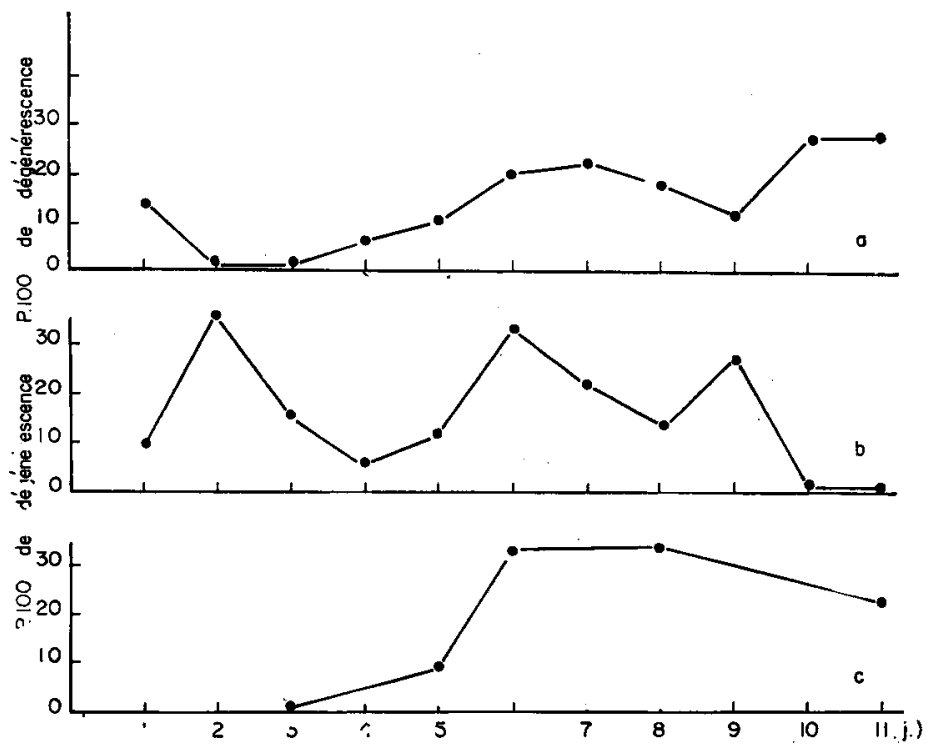

Fig. ro. - Pourcentages de dégénérescence :

a) œeufs " témoins ",

b) cufs " superovulés ",

c) œufs après traitement de $20 \mathrm{mg}$ de progestérone.

Après traitement à la progestérone et ovulation simple, la figure to $c$ indique le passage par une même période critique vers 6 jours et un taux de dégénérescence qui reste élevé jusqu'à II jours.

Dans les cas de superovulation combinée avec traitements à la progestérone, les courbes de dégénérescence ont un aspect assez analogue.

\section{DISCUSSION}

Ces études laissent apparaître la constance relative du développement embryonnaire pendant les 7 premiers jours. La segmentation des œufs parvenus dans l'utérus se poursuit jusqu'au $7^{\mathrm{e}}$ jour sans que l'apport de progestérone endogène ou exogène modifiant le rythme de descente dans l'oviducte, puisse en changer le cours. 
C'est à partir du $8^{\mathrm{e}}$ jour que le blastocyste devient sensible à l'équilibre hormonal maternel. Cette étape de son développement correspond à un stade physiologique. Il perd alors sa membrane pellucide, les cellules endodermiques commencent à émigrer du bouton embryonnaire vers le trophoblaste et la vitesse de segmentation subit une très nette accélération. Si on augmente le taux de progestérone chez les Brebis " témoins " ou si on se place dans les conditions de progestéronémie élevée (superovulation) le rythme des divisions est accéléré de façon spectaculaire. Comment la progestérone peut-elle provoquer une telle stimulation?

On peut envisager une action directe. La présence de progestérone dans les sécrétions de l'utérus n'a pas été démontrée actuellement chez la Brebis. Chez le Rat recevant des injections de progestérone ${ }_{14} \mathrm{C}$, un très faible marquage apparaît dans l'épithélium glandulaire (Rogers, Thomas, Yates, I965). Par ailleurs, 1'addition de progestérone au'milieu de culture des œufs de Souris (WHITTEN, I957) et de Lapine (DANIEI et LEVY, I964), s'est révélée arrêter les mitoses. I1 y a donc peu de chance pour que ce circuit direct se réalise, et s'il existe, favorise la croissance.

L'action indirecte de la progestérone semble plus vraisemblable. On connaît les variations cycliques de la composition des sécrétions de la muqueuse utérine chez le Rat, la Lapine, la Brebis et la Vache (HEAP, I962 ; HEAP et Lammings, I962). Chez la Lapine, après castration, les traitements progestogènes, non seulement restaurent la même teneur en acides aminés, des sécrétions utérines que chez les femelles cycliques, mais élèvent notablement la concentration en glycine et en sérine (GRÉGoIRE, GongSAKDI, RAKOFF, I96I), DANIEI, (I965) a trouvé une action stimulante de la glycine et de la sérine sur la croissance in vitro du blastocyste de Lapine de 5 jours.

On peut imaginer chez la Brebis une action analogue de la progestérone, augmentant dans les sécrétions utérines la concentration de certains amino-acides, qui stimuleraient le développement des blastocystes. Chez le Rat, une action hypertensive exercée par la progestérone et la meilleure irrigation de 1'utérus qui en résulte (CESsION-FossIon, LIMET, I965) a été identifiée au niveau du myomètre. Si un phénomène semblable se produisait chez la Brebis, 1'apport accéléré de métabolites pourrait également intervenir dans la modification du rythme desegmentation des blastocystes.

Cependant, la proportionnalité qui s'établit entre la taille du blastocyste et le taux de progestérone est limitée. En effet, la courbe de croissance des blastocystes de Lapine, établie en fonction de la quantité de progestérone administrée, trouvée par $\mathrm{Wu}$, se termine en palier pour la dose de $I \mathrm{mg} / \mathrm{j}$.

Bien qu'il s'agisse dans nos expériences d'un apport supplémentaire en présence de corps jaunes fonctionnels, nos résultats sont cependant comparables.

Notre raisonnement suppose que l'apport exogène de progestérone peut être identifié à la sécrétion d'un nombre correspondant de corps jaunes assurant le même taux de progestérone dans 1'utérus. Cette équivalence semble être démontrée par les expériences de castration pendant la gestation et de maintien des embryons par traitement progestéronique (PINCUS et WERTHESSEN, I938 ; CORNER, I928; ADAMS, I958; WU, rg6r ; HAFEZ, I964 : chez la Lapine ; CANIVENC et LAFFARGUE, I957; CarPent, I962 : chez la Ratte ; Meites, Webster, Young, Thord et Hatch, I95I : chez la Chèvre ; REASIDE et TURNER, I950 : chez la Vache). Cependant, Foote, Gooch, PoPE et CASIDA (I957) ont amélioré très nettement la survie embryonnaire chez les Brebis ovariectomisées, en accompagnant le traitement progestéronique d'œstrone. 
Peut-on imputer à la seule progestérone, l'action stimulante constatée?

En présence d'un ou de deux corps jaunes, un traitement de $20 \mathrm{mg}$ de progestérone accélère significativement le développement des blastocystes, mais dans des proportions toutefois bien inférieures à ce qu'elles sont avec 5 corps jaunes ou plus. L'ovaire secréterait donc, en plus de la progestérone, des stéroïdes capables de renforcer de façon très sensible l'effet constaté en présence de progestérone seule.

\section{Reçu pour publication en septembre 1967.}

\section{SUMMARY}

\section{EFFECT OF PROGESTERONE AND OVARIAN STERO DS}

ON EGG SEGMENTATION IN THE EWE

We have studied the egg segmentation rate of ewes killed I-I I days after ovulation. After fixation, embedment, and coloration, the nuclei were counted in the histological, seriated sections. The growth rate has also been studied in superovulated eggs (which contain more ovarian steroids and progesterone than usual). We have also studied the eggs of ewes having one or more ovulations and receiving exogenous progesterone.

Egg segmentation usually accelerated at 8 days (fig. I), but is was found that from days $\mathbf{I}$ to 7 the segmentation rate was the same in every case (fig. $1,4,7,8 b-c-d$ ). Thus, the period from 8 days onward was most strongly influenced by the action of superovulation which increased the segmentation rate (fig. 4). After progesterone treatment during normal ovulation, the segmentation rate also increased (fig. 7), but less than during superovulation. Protesterone treatment during superovulation slowed down the segmentation rate observed during superovulation (fig. $8 b-c-d)$.

\section{RÉFÉRENCES BIBLIOGRAPHIQUES}

ADAMS C. E., 1958. Egg development in the rabbit : the influence of post-coital ligation of the uterine tube and of ovariectomy. J. Endocr., 16, 283-293.

Canivenc R., Laffargue M., I957. Relation des corps jaunes et des blastocystes au cours de la nidation différée du Blaireau européen (Meles meles). C. r. Séanc. Soc. Biol., 151, 561-564.

Carpent G., I962. Le déséquilibre hormonal gravidique et ses répercussions sur la morphologie du foetus chez le Rat. Archs. Anat. micros. Morphol. exp., 51, 459-540.

Cession G., Cession-Fossion A., Limet R., 1965. Action de la progestérone sur trois agents endogènes à action hypertensive. C. r. Séanc. Soc. Biol., 159, 1864-1 866.

Corner G. W., I928 b. Physiology of the corpus luteum. I. - The effect of very early ablation of the corpus luteum upon embryos and uterus. Amer. J. Physiol., 86, 74-81.

DANIEL J. C. Jr., 1965. Studies on the growth of 5-day-old rabbit blastocysts in vitro. J. Embryol. exp. Morph., 18, 83-95.

DANIEL J. C. Jr., Levy J. D., I964. Action of progesterone as a cleavage inhibitor of rabbit ova in vitro. J. Reprod. Fert., 7, 323-329.

Davidson O. W., Schuchner E. B., WAdA K., 1965. Effect of clomiphene on rat zygotes. Fert. Steril., 16, 495-50r.

Foote W. D., Gooch L. D., Pope A. L., I957. The maintenance of early pregnancy in the ovariectomized ewe by injection of ovarian hormones. J. anim. Sci., 16, 986-990.

GREenwald G. S., 1957. Interruption of pregnancy in the rabbit by the administration of estrogen. $J$. exp. Zool., 185, 46I-482.

GREENWALD G. S., I958. Endocrine regulation of the secretion of mucin in the tubal epithelium of the rabbit. Anat. Rec., 130, 477-496.

Greenwald G. S., I962. The role of the mucin layer in development of the rabbit blastocyst. Anat. Rec., 148, 407-4I 6 . 
Gregoire ,A. T., Gongsakdi D., Rakoff M. D., I96r. Free amino acid content of the female rabbit genital tract. Fert. Steril, 12, 322-327.

HAFEZ E.S. E., 1964. Growth and survival of blastocysts in the domestic rabbit. II, -- Quantitative effects of exogenous progesterone following ovariectomy. J. Reprod. Fert., 7, 24 I-249.

HEAP R. B., I 962 . Sorne chemical constituents of uterine washings : a method of analysis with results from various species. J. Endocr., 24, 367-378.

Heap R. B., Lammings G. R., I962. The influence of ovarian hormones on some chemical constituents of the uterine washing of the rat and rabbit. J. Endocr., 25, 57-68.

Meites J., Webster H. D., Young F. W. et al., i95I. Effects of corpora lutea removal and replacement with progesterone on pregnancy in goats. J. Anim. Sci., 10, 410-416.

Pincus G., Werthessen N. T., I938. The maintenance of embryo life in ovariectomized rabbits. $A m$. J. Physiol., 124, 484-490.

ReAside J. I., TURner C. W., I950. A preliminary report on the role of progesterone in the maintenance of pregnancy in the cow. J. Dairy Sci., 33, 382 .

Rogers A. W., Thomas G. H., Yates K. M., 1965. Autoradiographic studies on the distribution of labelled progesterone in the uterus of the rat. Expl. Cell. Res., 40, 668-670.

SHort R. V., I961. Progesterone. Hormones in blood. Gray, Bacharach Ed., Acad. Press., N. Y., $379-437$.

Stormshak F., Inskeep E. K., Lynn J. E. et al., ı963. Progesterone levels in corpora lutea and ovarian effluent blood of the ewe. J. Anim. Sci., 22, 102 1-1026.

WhitTen W. K., I957. The effect of progesterone on the development of mouse eggs in vitro. J. Endocr., $16,80-85$.

Wintenberger S., I953. Recherches sur les relations entre l'œuf et le tractus maternel pendant les premiers stades du développement chez les Mammifères. Ann. Zoolech., 269-273.

Wintenberger-Torres S., ig6r. Mouvements des trompes et progression des ceufs chez la Brebis. Ann. Biol. anim. Bioch. Biophys., 1, 12 I-1 33.

WU D. H., I96r. Maintenance of pregnancy in castrated rabbits by an orally active progestational agent : $6 \propto$ methyl $17 \propto$ hydroxyprogesterone acetate. Fert. Steril., 12, 236-244. 